\title{
Evaluation of metabolic and immunological changes in streptozotocin-nicotinamide induced diabetic rats
}

\begin{abstract}
Type 2 diabetes is a chronic disease with growing public health concern globally. Finding remedies to assist this health issue requires recruiting appropriate animal model for experimental studies. This study was designated to evaluate metabolic and immunologic changes in streptozotocin-nicotinamide induced diabetic rats as a model of type 2 diabetes. Male rats were induced diabetes using nicotinamide $(110 \mathrm{mg} / \mathrm{kg})$ and streptozotocin (65 $\mathrm{mg} / \mathrm{kg}$ ). Following 42 days, biochemical and immunological tests showed that diabetic rats had higher levels of blood glucose, WBC, certain abnormalities in lipid profile and insufficient mitogenic responses of lymphocytes $(\mathrm{p}<0.05)$. However, the status of the total antioxidant, inflammatory biomarkers and other parameters of full blood count (except HCT) were not significantly altered. Phenotyping assay indicated insignificant lymphocyte subtype imbalances excluding a significant rise in the level of CD4+CD25+ marker $(p<0.05)$. This model of diabetic animals may represent some but not all symptoms of human type 2 diabetes.
\end{abstract}

Keyword: Streptozotocin; Nicotinamide; Type 2 diabetes; Immune system; Lymphocyte 